\title{
Dissatisfaction with maintenance of certification in academic pediatrics
}

\author{
Kate G. Ackerman ${ }^{1,2}$, Brendan Lee ${ }^{3}$ and Jake A. Kushner ${ }^{4}$
}

M edical science is increasingly complex, and efforts to utilize this knowledge in the most cost-effective, efficient, and evidenced-based manner is paramount to delivering the best health care for our patients and families. Pediatricians traditionally obtain board certification through the American Board of Pediatrics (ABP), one of the organizations under the American Board of Medical Specialties (ABMS). In the past $15 \mathrm{y}$, the $\mathrm{ABP}$ and other boards under the ABMS have expanded their scope, and this now includes a requirement to complete activities under a program trademarked as maintenance of certification (ABMS MOC). The expansion of rigorous certification requirements comes from a desire for the boards to assure the public that physicians have demonstrated a commitment to lifelong learning and professional development. A mandate from the public to the medical specialty boards for these changes is not apparent; thus, the justification for the MOC requirements that have been added to existing State Board requirements is unclear. Now, $12 \mathrm{y}$ after the $\mathrm{ABP}$ initiated MOC (with a redesign $9 \mathrm{y}$ ago), questions about efficacy and value of the ABMS MOC programs have surfaced as to its effectiveness in achieving the ABMS mission. Dissatisfaction with the American Board of Internal Medicine MOC has fueled a wide-scale discussion $(1,2)$ about whether this trademarked MOC system violates antitrust laws (3), whether management of conflict of interest is adequate, and whether there is adequate accountability and justification for the cost (4) and value of the required MOC activities. While any mandatory certification or regulatory process would be expected to be associated with some level of dissatisfaction, the growing controversy within multiple physician groups suggests that major changes should be made.

Who should evaluate the ABP MOC program? Are the regulations both cost and time effective? Since the ABP's customer is the public, and the public does not understand MOC, it is unclear whether adequate checks and balances are in place to assure that the system is working. The medical boards are trying to educate the public about the value of what they do. For example, at the time of this writing, the $\mathrm{ABP}$ website states, "although $\mathrm{ABP}$ certification is voluntary, nearly all qualified pediatricians seek this recognition" and the ABMS campaign,

Certification Matters, states that physicians voluntarily seek a rigorous MOC process. In fact, these activities are not voluntary for most academic pediatricians due to restrictions from employers, hospitals, and insurance companies. To gain insight into one group of physician opinions, the Society for Pediatric Research and the American Pediatric Society initiated a member survey. The societies have selective membership criteria and represent those doing high-quality research in child health and/or those who have developed national recognition and leadership skills. The societies also represent physicians who currently work for, have worked for, or who have received previous compensation from the ABP. The survey was done online and was designed to solicit all (both positive and negative) opinions about specific aspects of the MOC program within the pediatric academic community. Responses were collected over 3 wk in February/March of 2015. Of 3,769 survey links sent, there were 1,584 responses, with some nonresponders contacting the Society for Pediatric Research and the American Pediatric Society outside of the survey to indicate that MOC was not relevant to them. There were no monetary of other incentives given for completing the survey. Responses were collected from professionals associated with the Society for Pediatric Research (85\% of respondents), American Academy of Pediatrics (69\%), ABP (57\%), American Pediatric Society (45\%), Academic Pediatric Association (14.2\%), Association of Pediatric Program Directors (8.8\%), and the Association of Medical School Pediatric Department Chairs (4.9\%). These numbers reflect frequent affiliation with more than one academic society. Not surprisingly, the respondents were skewed toward more senior clinicians and/or clinician scientists with $51 \%$ professors, $27 \%$ associate professors, $13 \%$ assistant professors, and $9 \%$ instructors and fellows. At least $28 \%$ identified themselves as being in significant leadership positions. The group was largely subspecialty focused with $84 \%$ actively practicing a subspecialty of pediatrics and $16 \%$ practicing general pediatrics. There were more than 2,500 individual comments submitted from the membership about MOC, and the survey solicited responses to questions based on standard Likert scales. A review of the responses revealed three major themes: dissatisfaction in the choice of activities, lack of

'Department of Pediatrics, University of Rochester, Rochester, New York; ${ }^{2}$ Department of Biomedical Genetics, University of Rochester, Rochester, New York; ${ }^{3}$ Department of Molecular and Human Genetics, Baylor College of Medicine, Texas Children's Hospital, Houston, Texas; ${ }^{4}$ McNair Medical Institute, Pediatric Diabetes and Endocrinology, Baylor College of Medicine, Texas Children's Hospital, Houston, Texas. Correspondence: Kate G. Ackerman (kate_ackerman@urmc.rochester.edu)

Received 31 July 2015; accepted 9 September 2015; advance online publication 14 October 2015. doi:10.1038/pr.2015.189 
cost effectiveness (poor value of MOC), and a perception that the MOC activities interfere with other more important and meaningful activities.

Since it is important that the critique of MOC involve a widespread discussion of diplomates and stake holders, the deidentified data collected have been made publically available (Supplementary Data online). This will provide the medical community with a rich source of opinions and suggestions about how to move forward toward our unified goals of providing excellent health care of children for years to come.

\section{CHOICE OF ACTIVITIES}

There is dissatisfaction with the choice of activities available for MOC credit. The ABP Part 2 MOC, "Lifelong learning self assessment," requires completion of medical education activities, but the choice of activities feels restrictive to many and includes long lists of products that take time to sort through to identify matches for personal relevance and ideal cost. Questions about choice of activities were answered on a 5 -point scale (highly satisfied to not at all satisfied). Only $6 \%$ of respondents were "highly satisfied," whereas $26 \%$ were "not at all satisfied." Many subspecialists have become increasingly focused on a specific area of a subspecialty, perhaps a reasonable adaptation to the rapid doubling of medical information. This new breed of focused subspecialty clinician needs optimal flexibility in selecting Continuing Medical Education (CME) activities. While some Part 2 activities are valuable, a common sentiment was that there is no justification for not crediting a wide variety of activities that are required of an academic pediatrician. These include attendance at national meetings, publishing state-of-the-art articles about clinical topics, delivering or attending grand rounds, teaching clinical management to pediatric trainees, delivering or attending journal clubs, and participation in pediatric resuscitation teaching programs. The $\mathrm{ABP}$ ascertains that these other CME activities are expected by State Medical Boards, and therefore, credit is received within Part 1 MOC. The justification for this complexity is difficult to understand when the Part 2 MOC activities lack relevance to the clinical practice of many diplomates.

Satisfaction with choice of activities was worse for Part 4 of ABP MOC, "Performance in practice". Only 1.5\% were "highly satisfied," whereas 51\% were "not at all satisfied." Comments about choice were mainly focused on the redundancy and lack of credit given to institutionally implemented activities and the lack of activities that are relevant to specific clinical practice situations. The ABP has responded to some of these concerns with efforts to broaden the scope of acceptable activities within Part 4. Some institutions are now "Portfolio Sponsors" with authority to approve quality improvement (QI) projects within an institution or organization. The American Academy of Pediatrics, for example, is an MOC portfolio sponsor. There is now a mechanism to have individual QI projects and publications accepted as credit, although this involves an application fee, and the project may not be accepted since the criteria set by the ABP may not match the design of the project. The oversight of these activities by the $\mathrm{ABP}$ has been inefficient in creating or crediting adequate choice of meaningful activities for a diverse group of diplomates.

Perhaps, most important, there is no evidence that doing MOC Part 4 activities leads to better clinical performance. While some QI projects may improve the process of health care, there is no justification for requiring every diplomate to participate. Sound methods to study the impact of Part 4 have not been developed, and these activities are not currently justified as a mandatory component of an MOC portfolio.

\section{LACK OF COST EFFECTIVENESS (POOR VALUE OF MOC)}

The price of MOC activities varies between medical specialties under the ABMS. In pediatrics, 2015 fees are \$1,304 for MOC re-enrollment (includes one exam attempt), \$1,499 for MOC re-enrollment if one needs to regain certification, and $\$ 1,092$ per additional MOC examination. Additional fees apply for individual activities, and MOC portfolio sponsors and vendors, including the American Academy of Pediatrics, generate income from these activities. Fees for Part 4 include processing and application fees required to obtain individual or institutional approval. The cost satisfaction for Part 2, 3, and 4 activities was solicited on a 5-point scale (highly satisfied, satisfied, partially satisfied, not satisfied, and not at all satisfied). Most respondents were "not at all satisfied" with the cost of MOC. There was higher cost satisfaction with Part 2 than Part 3 or Part 4. For Part 4, 74\% were "not at all satisfied" or "not satisfied," whereas only $13 \%$ were "satisfied" or "highly satisfied." The cost of exams (Part 3) was "not satisfactory" for 69\% (bottom two choices) and "satisfactory" (top two choices) for $12 \%$. Costs of Part 2 were "not satisfactory" to $55 \%$ and "satisfactory" to $24 \%$.

The costs for MOC include the value of time spent doing MOC activities and the extra dollars spent paying for activities. While the former costs were recently estimated for those in Internal Medicine specialties (4), a reference is not yet available for pediatrics. Most of other dollars spent to cover MOC costs came from personal accounts. Only a minority (less than 19\%) had full institutional coverage for MOC expenses. Another $32 \%$ had costs covered or partially covered by educational allowances. These costs, of course, are added to the financial commitment of being a physician, which includes the costs of self-selected CME activities, state license fees, drug enforcement agency fees, money spent to attend conferences, and other activities and licensures necessary for practicing medicine. These activities occur independent of MOC; however, they are associated with MOC for fulfillment of Part 1. In addition to required fees for MOC participation, there are hidden costs (paid to third parties) for the educational materials and conferences many diplomates feel are necessary to prepare for secure recertification exams. These "hidden costs" can far exceed an educational allowance, and this displaces other educational needs. It is likely that the dissatisfaction with cost comes from money spent on the entire "system," not just from dollars paid to the ABP. The ABP is blamed for these costs, however, since they have helped to create a system where it is not possible to opt out of MOC. The question then becomes 


\section{Editorial | Ackerman et al.}

whether the system is well justified and whether time spent completing the MOC-specific, ABP-regulated activities adds value to clinical care and the practice of medicine. Finally, as MOC activities constitute an area of revenue growth, it raises inherent conflict of interest considerations for organizations that financially benefit from the expansion of MOC.

\section{THE PERCEPTION THAT THE MOC ACTIVITIES ARE INTERFERING WITH OTHER MORE IMPORTANT AND MEANINGFUL ACTIVITIES}

MOC activities require a significant investment of time and money for a physician, and it is essential that the activities help one maintain clinical competency and provide excellent patient care. Unfortunately, the perception is that the current MOC system is not meeting these stated goals. Many more felt that the Part 2, 3, and 4 activities were "unimportant" or "of little importance" for these goals (45-78\%) than those who felt that the activities were "very important" or "important" (10-31\%). Performance in practice (Part 4) was felt to be of extremely limited value with $78 \%$ feeling that the activities were unimportant/of little importance for providing excellent care, and only $10 \%$ deeming the activities of high value.

There is a high level of concern that the MOC activities deemed to be of low value in maintaining clinical excellence are taking away from other more important activities. Concerns are voiced both from those who are completing the activities and from "grandfathered" leaders in pediatrics who feel that the tasks are distracting from other important work. There are concerns that the sudden demand for QI projects from physicians not trained in the field and not dedicated to the field will confuse and dilute the substantial and important QI projects done by those who are specializing in this area. The design and implementation of a new project takes expertise and training while tagging onto a predesigned study often does not fit one's individual practice pattern.

\section{A PATH FORWARD}

Academic pediatricians are invested in improving the clinical care of children, and they value high quality continued medical education. While knowledge of a broad clinical scope may be appropriate for initial board certification, this does not match the reality of long-term medical practice. Competent and professional adult learners must seek educational opportunities that are highly relevant to their needs. The costs of ineffective or irrelevant activities to our health care community is too high at this critical time when we must try to deliver better quality care at a reduced price, and the perceived lack of value of required "voluntary" activities has caused much skepticism within academic pediatrics. It is now necessary to have an open discussion about the best way to create a system by which our patient community can be assured that their pediatricians are maintaining appropriate knowledge and skills. This system would need to flex to rapidly changing clinical climates, would need to have defined accountability, and would require a formal system for management of potential conflicts of interest. Improvement of long-term board certification options would best evolve from healthy competition and debate involving many groups within our health care delivery system. Until then, we need to suspend mandatory participation in activities of questionable current value.

This article was written to facilitate discussions about improving the systems by which we maintain high standards in the clinical care of children. The article represents the views of the authors only and is not a statement from the Society for Pediatric Research, the American Pediatric Society, or the other organizations whose members participated in the survey.

\section{SUPPLEMENTARY MATERIAL}

Supplementary material is linked to the online version of the paper at http:// www.nature.com/pr

\section{ACKNOWLEDGMENTS}

The compiled survey results (Supplementary Data online to this article) have been previously published on the APS/SPR website. We thank Jana Wells and Eileen Fenton for compiling the data into a presentable format.

\section{STATEMENT OF FINANCIAL SUPPORT}

No grant funding or other financial support was received for this editorial.

Disclosure: The authors have no disclosures/conflicts of interest.

\section{REFERENCES}

1. Teirstein PS. Boarded to death-why maintenance of certification is bad for doctors and patients. N Engl J Med 2015;372:106-8.

2. Irons MB, Nora LM. Maintenance of certification 2.0-strong start, continued evolution. N Engl J Med 2015;372:104-6.

3. Association of American Physicians \& Surgeons, Inc. v. American Board of Medical Specialties, No. 13-2609 (PGS) (LHG). (http://aapsonline.org/ AAPSvABMSresponse-6-3-2014.pdf)

4. Sandhu AT, Dudley RA, Kazi DS. A cost analysis of the American Board of Internal Medicine's maintenance-of-certification program. Ann Intern Med 2015;163:401-8. 